\title{
Morphological instability in a directionally solidifying binary solution with an imposed shear flow
}

\author{
By C. A. CHUNG AND FALIN CHEN \\ Institute of Applied Mechanics, National Taiwan University, Taipei, Taiwan 10764, ROC \\ e-mail: falin@spring.iam.ntu.edu.tw
}

(Received 1 February 2000 and in revised form 29 August 2000)

The effect of an imposed shear flow on the stability of directionally solidifying binary alloys is investigated. It is shown that without the imposed shear flow the system is dominated by stationary boundary-layer-mode convection, a convection of saltfinger type confined to the solute boundary layer above the melt/mush interface. When the shear flow (no matter how small) is imposed, the boundary-layer mode becomes a longitudinal mode (roll-axis parallel to the imposed flow) propagating in the direction perpendicular to the shear flow, while the modes containing a transverse component are inhibited. As the shear flow becomes large enough, a transverse mode (roll-axis perpendicular to the imposed flow) of very unstable characteristics is induced. This mode, called the morphological mode, can exist even without buoyancy. It is triggered by the flow induced in the mushy layer through the Bernoulli force, a pressure variation resulting from the imposed flow passing along the corrugated melt/mush interface. It, nonetheless, has no relation to the boundary layer instability of the shear flow. The effect of imposed shear flow is so significant that the stability characteristics can be entirely different when the intensity of the imposed flow is larger than a critical value, which is calculated in the present paper.

\section{Introduction}

The mechanism driving the formation of freckles in directionally solidified binary alloys has attracted a great deal of research effort in the past several decades because of its catastrophic effects on the mechanical properties of cast alloys. Copley et al. (1970) illustrated experimentally that freckles are a direct result of plume convection, a buoyant flow passing through vertical channels in the dendritic mushy zone into the melt above. To date, several experimental and theoretical investigations (Tait \& Jaupart 1992; Chen, Lu \& Yang 1994; Amberg \& Homsy 1993; Anderson \& Worster 1995; Chung \& Chen 2000a) have shown that the plume convection is closely related to the convection in the mushy layer. To suppress the plume convection, Sample \& Hellawell (1984) suggested in their experiment that rotating the casting mode with respect to an inclined axis would result in a casting without freckles. Chung $\&$ Chen $(2000 b)$ found from a theoretical approach that, because of the inclined rotation, a strong shear flow occurs above the melt/mush interface, which might play a critical role in suppressing the freckles. This argument is partly supported by the stability analysis of $\mathrm{Lu} \&$ Chen (1997), who showed that a vertical rotation could not effectively inhibit the convection in the mush because no shear flow is induced. Nevertheless, as we will show in the present paper, under some circumstances this 
argument cannot be justified because an imposed parallel shear flow may induce a very unstable mode, which may in turn trigger the formation of plumes. We therefore conduct the present study to investigate this argument, to see how an imposed shear flow can influence the stability of convection in the directionally solidifying binary solution.

The effect of shear flow on directionally solidifying alloys has been investigated by many researchers, who focused mainly on the coupled convective and morphological instabilities. Delves $(1968,1971)$ first considered the effect of shear flow on the morphological instability, imposing quadratic and Blasius boundary-layer flows above the melt/solid interface. Coriell et al. (1984) extended the work of Delves by considering double-diffusive convection under the influence of plane Couette flow parallel to the melt/solid interface. All of them concluded that the shear flow inhibits the onset of the transverse mode, a two-dimensional roll with axis perpendicular to the flow direction, and the onset of the instability is always of the longitudinal mode, a two-dimensional roll with axis parallel to the flow direction. Their findings are similar to those found in both Rayleigh-Bénard convection (Gershuni \& Zhukhovitskii 1976) and salt-finger convection (Linden 1974; Chen \& Chen 1997). Later, MacFadden, Coriell \& Alexander (1988) imposed a plane stagnation flow vertically onto the melt/solid interface while focusing only on the stability of the transverse mode. More recently, Forth \& Wheeler $(1989,1992)$ have considered the effect of the asymptotic suction boundary-layer flow and showed from both numerical and analytical approaches that the suction-layer stability is generally unaffected by the freezing melt/solid interface. They showed also that a longitudinal travelling wave is always induced first by the shear flow. Davis and coworkers (see the review by Davis 1990) attacked this problem by emphasizing the instability of convection modes of long wavelength for some special cases. All these studies considered a system of bulk melt overlying a solid substrate.

A major difference of the present study from these previous investigations stems from the fact that in the present physical system there is a dendritic mushy layer (the mush) lying between the fluid layer (the melt) above and the solid substrate below. In such a system, by and large, two kinds of convection modes may occur (Worster 1992). One is the so-called boundary-layer mode, a salt-finger-type convection cell confined to the shallow solute layer above the melt/mush interface. The other is the so-called mushy-layer mode, a convection cell circulating largely in the mush. As we shall show in the present paper, an imposed shear flow, which has the velocity profile of a suction boundary-layer flow and moves in the direction parallel to the melt/mush interface, has a profound influence on the stability of the boundary-layer mode but has virtually no influence on the mushy-layer mode. More precisely, when the shear flow is imposed, the roll-axis of the salt-finger cell (boundary-layer mode) aligns immediately with the shear flow (becoming a longitudinal mode), similar to the result found previously by Coriell et al. (1984) that under the influence of the shear flow the longitudinal mode convection is the most unstable one. In some circumstances, however, the shear flow may induce a transverse mode, which will eventually dominate the system. This novel instability mode turns out to be a result of the interaction between the imposed shear flow and the deformation of the melt/mush interface, and has no direct connection with the buoyancy. We shall therefore call it the morphological mode in the present paper.

Since in the present system the melt/mush interface moves vertically upwards with a constant velocity, if we fix the coordinates on the interface, the fluid is seen to pass partially vertically downwards into the melt/mush interface, not unlike a boundary- 
layer flow under asymptotic suction into a porous medium below. Because buoyancy plays no role in the onset of the morphological mode, the possible analogy between the present system and the asymptotic-suction boundary layer flow (Hocking 1975) is examined, so that the relation between the morphological instability mode and the instability of the asymptotic-suction boundary layer can be clarified.

Several months after we finished the first phase of the stability analysis (Chung 1998), similar work by Feltham \& Worster (1999) came to our attention. They investigated the morphological instability of a mushy layer unidirectionally solidifying from above, in which a parallel flow (inviscid or viscous) is imposed in the melt below the mush/melt interface. They indicated that the flow induced in the mush due to the Bernoulli effect along the incipient corrugation of the interface causes the morphological instability, and the imposed parallel flow plays a dominant role in translating the interface shape horizontally in the asymptotic regime considered. Owing to the similarity between the present system and that of Feltham \& Worster (1999), we investigate whether the present morphological mode is the same as the mode found by Feltham \& Worster (1999), and use our results to help understand the mechanism driving the occurrence of the novel mode.

In the following, we describe in $\S 2$ the physical configuration and the mathematical formulation of the problem considered. The basic-state solution is given in $\S 3$ and the formulation for the linear stability analysis is derived in $\S 4$. The effects of imposed shear flow on the stability are studied by the linear approach in $\S 5$. We also discuss in $\S 6$ the relevance of the present system to the case of the boundary-layer flow under asymptotic suction. A discussion on the mechanism triggering the morphological mode is given in $\S 7$. Finally conclusions are drawn in $\S 8$.

\section{Mathematical formulation}

Consider the system shown in figure 1 , a binary solution of concentration $C_{0}$ and temperature $T_{0}$ solidifying from below. A dendritic mushy layer lies between the semiinfinite fluid layer above and the solid layer of eutectic temperature $T_{E}$ and eutectic concentration $C_{E}$ below. We assume that the mush/solid interface grows upwards with a constant velocity $V$ and employ a Galilean transformation with respect to $V$, fixing the Cartesian coordinate on the mush/solid interface. Accordingly, $z=0$ accounts for the position of the mush/solid interface and $z=h(x, y, t)$ for the position of the melt/mush interface, which is unknown beforehand. It is assumed that the physical domain is horizontally infinite so that in the far field a driving flow $\boldsymbol{u}(z \rightarrow \infty)=U_{\infty} \hat{\boldsymbol{e}}_{x}$ parallel to the melt/mush interface can be imposed, where $\hat{\boldsymbol{e}}_{x}$ is the unit vector in $\boldsymbol{x}$-direction.

We consider the melt as a Newtonian fluid, the mushy layer as an isotropic porous medium, and apply the Boussinesq approximation. To simplify the equations we assume that the specific heat and the thermal conductivity of the solid are the same as their liquid-phase counterparts (Worster 1986) and assume that thermodynamic equilibrium condition holds in the mushy layer. Specifically, the temperature and concentration in the mush satisfy the liquidus relation

$$
T-T_{L}\left(C_{0}\right)=\Gamma\left(C-C_{0}\right),
$$

where the liquidus is assumed to be linear and $T_{L}\left(C_{0}\right)$ is the liquidus temperature corresponding to the concentration $C_{0}$. This condition has been confirmed valid for ammonium chloride solution by Chen et al. (1994), and is believed to be also valid for other binary solutions of similar physical characteristics. 


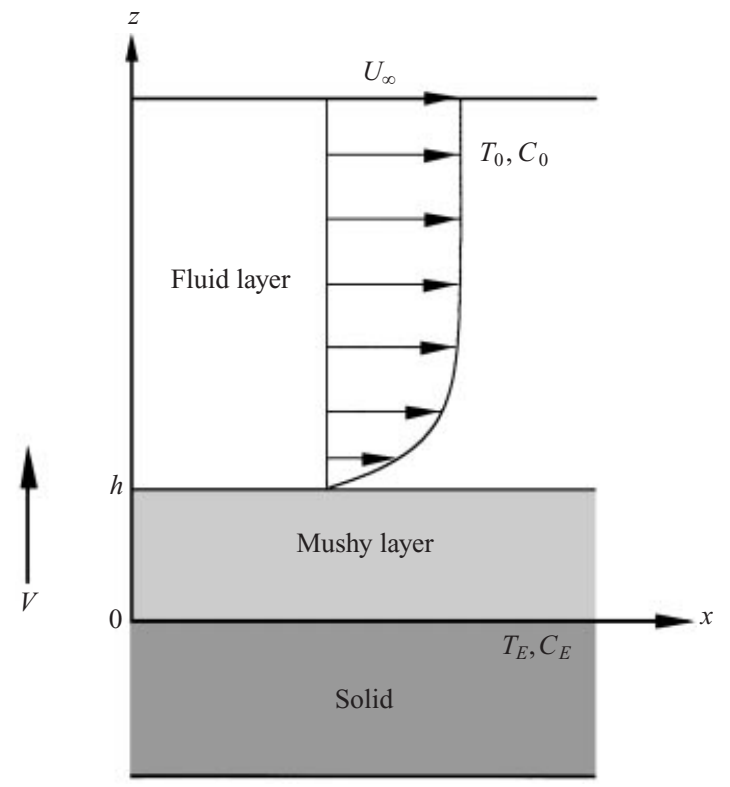

FIGURE 1. A schematic of the physical system considered. A shear flow is imposed on a binary solution which is directionally solidifying from below. The coordinates are fixed on the mush/solid interface, which moves upwards with a constant velocity $V$.

We non-dimensionalize the governing equations with the following scales: $V$ for velocity, $H=\kappa / V$ for length, $\kappa / V^{2}$ for time, and $\bar{\beta} \Delta C \rho_{0} g \kappa / V$ for pressure, where $\kappa$ is the thermal diffusivity, $\bar{\beta}=\beta^{*}-\Gamma \alpha^{*}, \alpha^{*}$ and $\beta^{*}$ are respectively the thermal and concentration expansion coefficients, $\Delta C=C_{0}-C_{E}, \rho_{0}$ is the reference density corresponding to concentration $C_{0}$ and temperature $T_{L}\left(C_{0}\right)$, and $g$ is the gravitational acceleration. The dimensionless temperature and concentration are, respectively,

$$
\theta=\frac{T-T_{L}\left(C_{0}\right)}{\Delta T}, \quad \Theta=\frac{C-C_{0}}{\Delta C},
$$

where $\Delta T=\Gamma \Delta C=T_{L}\left(C_{0}\right)-T_{E}$.

To describe the fluid motion of the system, two sets of governing equations are required: one for the fluid layer and one for the mushy layer. The governing equations of the fluid layer are

$$
\begin{gathered}
\nabla \cdot \boldsymbol{u}=0 \\
\frac{1}{\sigma}\left[\frac{\partial}{\partial t}-\frac{\partial}{\partial z}+\boldsymbol{u} \cdot \nabla\right] \boldsymbol{u}=\nabla^{2} \boldsymbol{u}-\mathscr{H} R_{m} \nabla p+\left(R_{t} \theta-R_{c} \Theta\right) \hat{\boldsymbol{e}}_{z} \\
\left(\frac{\partial}{\partial t}-\frac{\partial}{\partial z}+\boldsymbol{u} \cdot \nabla\right) \theta=\nabla^{2} \theta \\
\left(\frac{\partial}{\partial t}-\frac{\partial}{\partial z}+\boldsymbol{u} \cdot \nabla\right) \Theta=\varepsilon \nabla^{2} \Theta
\end{gathered}
$$

and those of the mushy layer are

$$
\begin{gathered}
\nabla \cdot \boldsymbol{u}=0, \\
\frac{\boldsymbol{u}}{\Pi(\chi)}=-R_{m} \nabla p-R_{m} \theta \hat{\boldsymbol{e}}_{z},
\end{gathered}
$$




$$
\begin{gathered}
\left(\frac{\partial}{\partial t}-\frac{\partial}{\partial z}+\boldsymbol{u} \cdot \nabla\right) \theta=\nabla^{2} \theta-\mathscr{F}\left(\frac{\partial}{\partial t}-\frac{\partial}{\partial z}\right) \chi, \\
\chi\left(\frac{\partial}{\partial t}-\frac{\partial}{\partial z}\right) \theta+\boldsymbol{u} \cdot \nabla \theta=-(\theta-\mathscr{C})\left(\frac{\partial}{\partial t}-\frac{\partial}{\partial z}\right) \chi,
\end{gathered}
$$

where $R_{t}$ and $R_{c}$ are respectively the thermal and solute Rayleigh numbers of the fluid layer and $R_{m}$ is the Rayleigh number of the mushy layer. They are defined as

$$
R_{t} \equiv \frac{g \alpha^{*} \Delta T H^{3}}{\kappa v}, \quad R_{c} \equiv \frac{g \beta^{*} \Delta C H^{3}}{\kappa v}, \quad R_{m} \equiv \frac{g \bar{\beta} \Delta C \Pi_{0} H}{\kappa v},
$$

in which $v$ is the kinematic viscosity of the fluid and $\Pi_{0}$ is the reference permeability of the mush, assumed to be $\Pi_{0}=\Pi(1)$. It is noted that $R_{t}, R_{c}$ and $R_{m}$ satisfy the relations $R_{t}=\mathscr{A} \mathscr{H} R_{m}, R_{c}=(1+\mathscr{A}) \mathscr{H} R_{m}$, where $\mathscr{H}$ is the inverse Darcy number defined as $\mathscr{H} \equiv H^{2} / \Pi_{0}$, and $\mathscr{A}=\Gamma \alpha^{*} / \bar{\beta}$ is the buoyancy ratio. In addition, $\varepsilon \equiv D_{f} / \kappa$ is the inverse Lewis number, $D_{f}$ the mass diffusivity of the fluid, $\sigma \equiv v / \kappa$ the Prandt number, $\mathscr{F} \equiv \mathscr{L} /(c \Delta T)$ the Stefan number, $c$ the specific heat per unit volume, $\mathscr{C} \equiv\left(C_{s}-C_{0}\right) / \Delta C$ the concentration ratio, $\mathscr{L}$ the latent heat per unit volume, and $C_{s}$ the concentration of the solid. It is noted that the value of $\varepsilon$ for the mush is very small. We thus neglect the mass diffusion in the mush. Because of the thermodynamic equilibrium condition, the dimensionless liquidus relation in the mush can be written as $\theta=\Theta$, which has been introduced into $(4 b)$ and $(4 d)$.

For the boundary conditions at the far field $z \rightarrow \infty$ we have

$$
\theta \rightarrow \theta_{0}, \quad \Theta \rightarrow 0, \quad \boldsymbol{u} \rightarrow U_{\infty} \hat{\boldsymbol{e}}_{x}
$$

At the melt/mush interface $z=h(x, y, t)$ we have

$$
\begin{gathered}
\theta=\Theta, \quad \frac{\partial \theta}{\partial n}=\frac{\partial \Theta}{\partial n}, \quad[\boldsymbol{u} \cdot \boldsymbol{n}]=0, \quad[\theta]=0 \\
{\left[\frac{\partial \theta}{\partial n}\right]=0, \quad \chi=1, \quad\left[\sigma_{n}\right]=0,\left.\quad \frac{\partial u_{2}}{\partial n}\right|_{h^{+}}=\Lambda \sqrt{\frac{\mathscr{H}}{\Pi(1)}}\left(\left.u_{2}\right|_{h^{+}}-\left.u_{2}\right|_{h^{-}}\right),}
\end{gathered}
$$

in which [] denotes the jump of the enclosed quantity across the interface, $(7 a)$ is the continuity of concentration, $(7 b)$ is the marginal equilibrium condition proposed by Worster (1986), $(7 c)$ is the continuity of mass flux, $(7 d)$ is the continuity of temperature, $(7 e)$ is the continuity of heat flux resulting from $(7 f)$ and the Stefan condition, $(7 f)$ illustrates that the liquid fraction of the mush at the interface is equal to unity, $(7 \mathrm{~g})$ accounts for the continuity of the normal stress, and $(7 h)$ is an empirical condition proposed by Beavers \& Joseph (1967), where $\boldsymbol{u}_{2}$ is the planar velocity and $\Lambda$ is an empirical constant determined by experiment. Finally, at the mush/solid interface $z=0$ we have

$$
\theta=-1, \quad \boldsymbol{u} \cdot \boldsymbol{n}=0 .
$$

It is noted that the present formulations, except $(6 c)$, are similar to those shown in Worster (1992) and Chen et al. (1994), in which a detailed discussion of the equations as well as the physical parameters are provided.

\section{Basic-state solutions}

Since the physical domain considered is assumed to be horizontally infinite, we seek a basic-state solution in which the dependent variables depend only on $z$. By 
applying the scale analysis of Chung \& Chen (2000b), we obtain the solutions in the fluid layer $h_{b}<z<\infty$ :

$$
\begin{gathered}
u_{b}=U_{\infty}\left\{1-\left(\frac{\Lambda \sqrt{\mathscr{H} / \Pi(1)}}{1 / \sigma+\Lambda \sqrt{\mathscr{H} / \Pi(1)}}\right) \exp \left[-\left(z-h_{b}\right) / \sigma\right]\right\}, \\
v_{b}=0, \quad w_{b}=0, \\
\theta_{b}=\theta_{0}+\left(\theta_{t}-\theta_{0}\right) \exp \left[-\left(z-h_{b}\right)\right], \\
\Theta_{b}=\theta_{i} \exp \left[-\left(\frac{z-h_{b}}{\varepsilon}\right)\right],
\end{gathered}
$$

in which $\theta_{i}=(-\varepsilon /(1-\varepsilon)) \theta_{0}$ is the temperature (or the concentration) at the melt/mush interface. In the mushy layer $0<z<h_{b}$, we obtain

$$
\begin{gathered}
u_{b}=0, \quad v_{b}=0, \quad w_{b}=0, \\
z=\frac{\alpha-\mathscr{C}}{\alpha-\beta} \ln \left(\frac{\alpha+1}{\alpha-\theta_{b}}\right)+\frac{\mathscr{C}-\beta}{\alpha-\beta} \ln \left(\frac{\beta+1}{\beta-\theta_{b}}\right) . \\
\chi_{b}=\frac{\mathscr{C}-\theta_{i}}{\mathscr{C}-\theta_{b}} .
\end{gathered}
$$

where $\alpha=A+B, \beta=A-B, A=\left(\mathscr{C}+\theta_{0}+\mathscr{F}\right) / 2$, and $B=\sqrt{A^{2}-\mathscr{C} \theta_{0}-\mathscr{F} \theta_{i}}$. The temperature in the mush as shown in (10d) is to be solved implicitly and the basic state porosity $\chi_{b}$ is a function of temperature. The height of the mushy layer $h_{b}$ can be obtained by substituting $\theta_{b}=\theta_{i}$ into $(10 d)$. Note that $(10 a-e)$ are the same as those shown by Worster (1992) and Chen et al. (1994).

To elucidate the physical sense of the basic-state solution, we consider the case of $26 \mathrm{wt} \%$ ammonium chloride solution, whose physical properties are listed in table 1. With these parameters the height of the mush is $h_{b}=0.991$, the fluid in the mush is essentially quiescent, and the flow in the fluid layer is a boundary-layer-type shear flow (figure 2). The characteristic length scale of the velocity variation of this shear flow is proportional to the Prandtl number $\sigma$. The flow velocity increases exponentially from virtually zero near the melt/mush interface to $U_{\infty}$ in the far field. The velocity near the interface can be obtained from $(10 a)$ as

$$
u_{b}=U_{\infty}\left(\frac{1 / \sigma}{1 / \sigma+\Lambda \sqrt{\mathscr{H} / \Pi(1)}}\right) \approx 0,
$$

which can therefore be taken as a non-slip condition on the interface, as considered by Feltham \& Worster (1999).

The results of (9) and (10) for the temperature and solute distributions are exactly the same as those of previous studies (Worster 1992; Chen et al. 1994), implying that neither the temperature nor concentration fields are affected by the presence of the shear flow. The temperature and the concentration in the mushy layer are the same, increasing exponentially with height. In the fluid layer, both the temperature and the concentration also increase exponentially with height. The characteristic length scale of the temperature variation is $O(1)$ while that of the concentration variation is $O(\varepsilon)$. Since $\varepsilon \ll 1$, the variation of concentration is confined to a shallow solutal boundary layer above the melt/mush interface. Beyond this layer, the solute concentration remains virtually constant. For the same reason, the interface temperature is very 


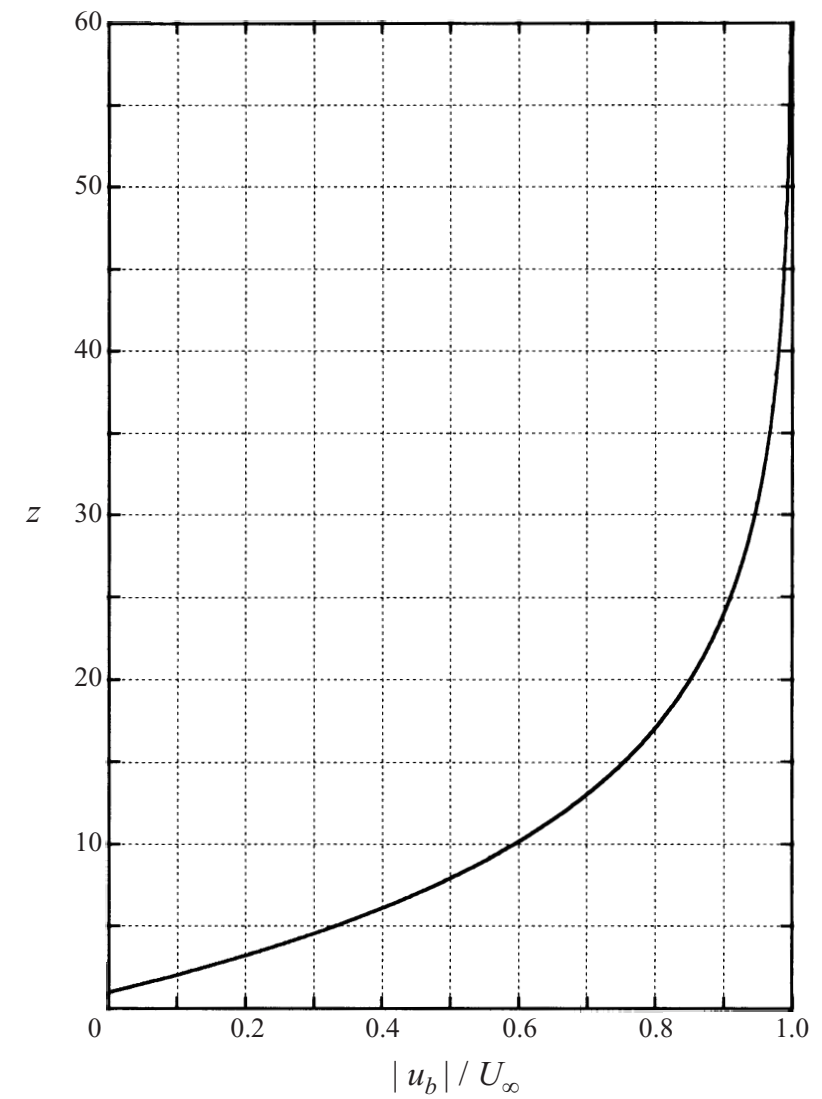

FigURE 2. The velocity profile of the imposed shear flow in a $26 \mathrm{wt} \%$ ammonium chloride solution. The melt/mush interface is at about $z=0.991$. The thickness of the shear boundary layer is about 50 times of the mushy-layer height, and is of a similar length scale as the wavelength of the suction-layer mode shown in $\S 6$. The imposition of $U_{\infty}$ at $z \rightarrow \infty$ will theoretically lead to the formation of a linear Couette flow while the present exponential velocity profile is a result of the combination of the Couette flow and the velocity $V$ of the freezing boundary, on which the coordinates are fixed.

close to the liquidus temperature $T_{L}\left(C_{0}\right)$, namely $\theta_{i} \approx 0$. The porosity in general is close to unity because $\mathscr{C} \gg 1$. The density distribution in both the mush and the solute boundary layer is potentially unstable to small disturbances while in most of the fluid layer it is statically stable.

\section{Linear stability analysis}

A linear stability analysis is employed to investigate the stability characteristics of the system described in $\S 2$. We add small perturbations to the basic state of $\S 3$ and substitute the combination into the governing equations, (3) and (4), and the associated boundary conditions (6)-(8). By neglecting the products of small quantities, we obtain the following linearized equations:

in the fluid layer

$$
\left(\frac{\partial}{\partial t}-\frac{\partial}{\partial z}+u_{b} \frac{\partial}{\partial x}-\nabla^{2}\right) \theta=-\theta_{b}^{\prime} w
$$




\begin{tabular}{llll}
\hline$\Gamma$ & $4.75{ }^{\circ} \mathrm{C} / \%$ & $\sigma$ & 10 \\
$\mathscr{L}$ & $338 \mathrm{~J} \mathrm{~cm} \mathrm{~cm}^{-3}$ & $\varepsilon$ & 0.013 \\
$D_{f}$ & $1.7 \times 10^{-5} \mathrm{~cm}^{2} \mathrm{~s}^{-1}$ & $\mathscr{A}$ & 0.65 \\
$v$ & $1.2 \times 10^{-2} \mathrm{~cm}^{2} \mathrm{~s}^{-1}$ & $\mathscr{F}$ & 3.2 \\
$\alpha^{*}$ & $3.832 \times 10^{-4} \mathrm{C}^{-1}$ & $\mathscr{C}$ & 12.3 \\
$\beta^{*}$ & $2.82 \times 10^{-3} / \%$ & $\mathscr{H}$ & $3.5 \times 10^{6}$ \\
$T_{E}$ & $-15.4^{\circ} \mathrm{C}$ & $\theta_{0}$ & 0.5 \\
$C_{E}$ & $19.7 \%$ & $\Lambda$ & 0.1 \\
$C_{s}$ & $100 \%$ & &
\end{tabular}

TABLE 1 . The physical parameters and the corresponding values for $26 \mathrm{wt} \%$ ammonium chloride solution.

$$
\begin{gathered}
\left(\frac{\partial}{\partial t}-\frac{\partial}{\partial z}+u_{b} \frac{\partial}{\partial x}-\varepsilon \nabla^{2}\right) \Theta=-\Theta_{b}^{\prime} w \\
\frac{1}{\sigma}\left(\frac{\partial}{\partial t}-\frac{\partial}{\partial z}+u_{b} \frac{\partial}{\partial x}-\sigma \nabla^{2}\right) \nabla^{2} w-\frac{1}{\sigma} u_{b}^{n} \frac{\partial w}{\partial x}=\nabla_{H}^{2}\left(R_{t} \theta-R_{c} \Theta\right)
\end{gathered}
$$

and in the mushy layer

$$
\begin{gathered}
\left(\frac{\partial}{\partial t}-\frac{\partial}{\partial z}-\nabla^{2}\right) \theta+\mathscr{F}\left(\frac{\partial}{\partial t}-\frac{\partial}{\partial z}\right) \chi=-\theta_{b}^{\prime} w \\
{\left[\chi_{b}\left(\frac{\partial}{\partial t}-\frac{\partial}{\partial z}\right)-\chi_{b}^{\prime}\right] \theta+\left[\left(\theta_{b}-C\right)\left(\frac{\partial}{\partial t}-\frac{\partial}{\partial z}\right)-\theta_{b}^{\prime}\right] \chi=-\theta_{b}^{\prime} w} \\
\nabla^{2} w=-R_{m} \nabla_{H}^{2} \theta .
\end{gathered}
$$

The boundary conditions at $z=0$ are

$$
\theta=0, \quad w=0,
$$

at $z=h_{b}$ are

$$
\begin{gathered}
\theta=\Theta, \quad \frac{\partial \Theta}{\partial z}-\frac{\partial \theta}{\partial z}=\left(\frac{1-\varepsilon}{\varepsilon}\right) \theta_{b}^{\prime} h, \quad[\theta]=0, \quad\left[\frac{\partial \theta}{\partial z}\right]=\left(\frac{\mathscr{F} \theta_{b}^{\prime}}{\theta_{i}-\mathscr{C}}\right) h \\
\chi=\left(\frac{\theta_{b}^{\prime}}{\theta_{i}-\mathscr{C}}\right) h, \quad[w]=u_{b+} \frac{\partial h}{\partial x} \\
\left.\frac{\partial^{2} w}{\partial z^{2}}\right|_{+}=\Lambda \sqrt{\frac{\mathscr{H}}{\Pi(1)}}\left(\left.\frac{\partial w}{\partial z}\right|_{+}-\left.\frac{\partial w}{\partial z}\right|_{-}-u_{b+}^{\prime} \frac{\partial h}{\partial x}\right)+u_{b+}^{\prime \prime} \frac{\partial h}{\partial x} \\
\left.\frac{\mathscr{H}}{\Pi(1)} \frac{\partial w}{\partial z}\right|_{-}=\left\{\left[\frac{1}{\sigma}\left(\frac{\partial}{\partial t}-\frac{\partial}{\partial z}+u_{b+} \frac{\partial}{\partial x}\right)-\frac{\partial^{2}}{\partial z^{2}}-3 \nabla_{H}^{2}\right] \frac{\partial}{\partial z}-\frac{u_{b}^{\prime}}{\sigma} \frac{\partial}{\partial x}\right\} w_{+} \\
+2 u_{b+}^{\prime} \frac{\partial}{\partial x}\left(\nabla_{H}^{2} h\right)
\end{gathered}
$$

and at $z \rightarrow \infty$ are

$$
\theta \rightarrow 0, \quad \Theta \rightarrow 0, \quad w \rightarrow 0, \quad \frac{\partial w}{\partial z} \rightarrow 0
$$

$(16 a-d)$ 
In these equations all the horizontal components of velocities and pressure have been eliminated and $\theta, \Theta, w$, and $\chi$ are now the disturbed quantities and $h=h(x, y, t)$ is the disturbance of the melt/mush interface. When deriving the perturbation equations we have assumed $\Pi=\Pi(1)$, as did by Worster (1992) and Chen et al. (1994).

By applying normal mode expansion

$$
\left[\begin{array}{l}
\theta(x, y, z, t) \\
\Theta(x, y, z, t) \\
w(x, y, z, t) \\
\chi(x, y, z, t) \\
h(x, y, t)
\end{array}\right]=\left[\begin{array}{l}
\hat{\theta}(z) \\
\hat{\Theta}(z) \\
\hat{w}(z) \\
\hat{\chi}(z) \\
\hat{h}
\end{array}\right] \exp \left[\omega t+\mathrm{i}\left(k_{x} x+k_{y} y\right)\right]+\text { c.c., }
$$

to (12)-(16), the partial differential equations are converted into ordinary differential equations. In (17) $\omega=\omega_{r}+\mathrm{i} \omega_{i}$ is complex, where $\omega_{r}$ is the growth rate of the disturbance and $\omega_{i}$ is the oscillation frequency, $k_{x}$ and $k_{y}$ are the wavenumbers of the disturbances along respectively the $\boldsymbol{x}$ - and $\boldsymbol{y}$-directions. If $\omega_{r}>0$ the basic state is unstable; if $\omega_{r}<0$ the basic state is stable; and when $\omega_{r}=0$ the basic state is neutral.

We employ Squire's transformation and define the horizontal wavenumber as $k=\sqrt{k_{x}^{2}+k_{y}^{2}}$ and the 'effective shear flow velocity' $\bar{u}_{b}$ as

$$
\bar{u}_{b} \equiv k_{x} u_{b} / k=U_{\infty}^{\prime}\left\{1-\left(\frac{\Lambda \sqrt{\mathscr{H} / \Pi(1)}}{1 / \sigma+\Lambda \sqrt{\mathscr{H} / \Pi(1)}}\right) \exp \left[-\left(z-h_{b}\right) / \sigma\right]\right\},
$$

in which $U_{\infty}^{\prime}$ is called the effective shear flow velocity coefficient, defined as

$$
U_{\infty}^{\prime} \equiv k_{x} U_{\infty} / k=U_{\infty} \cos \left(\phi_{k}\right),
$$

where $\phi_{k}$ is the angle between the shear flow and the propagation direction of the disturbance. In other words, the effective shear flow is the component of the shear flow in the direction of the propagation of the disturbance. The physical meaning of the parameter $U_{\infty}^{\prime}$ will be discussed in more detail in $\S 5$. The use of (18) and (19) results in a simplification of the equations as well as a reduction of the number of parameters by one.

The simplified equations in the fluid layer after Squire's transformation can be written as

$$
\begin{gathered}
\left(\mathrm{D}^{2}+\mathrm{D}-\mathrm{i} k \bar{u}_{b}-\omega-k^{2}\right) \hat{\theta}=\theta_{b}^{\prime} \hat{w}, \\
\left(\varepsilon \mathrm{D}^{2}+\mathrm{D}-\mathrm{i} k \bar{u}_{b}-\omega-\varepsilon k^{2}\right) \hat{\Theta}=\Theta_{b}^{\prime} \hat{w}, \\
{\left[\mathrm{D}^{2}+\frac{1}{\sigma}(\mathrm{D}-\omega)-k^{2}-\frac{\mathrm{i} k \bar{u}_{b}}{\sigma}\right]\left(\mathrm{D}^{2}-k^{2}\right) \hat{w}+\frac{\mathrm{i} k \bar{u}_{b}^{\prime \prime}}{\sigma} \hat{w}=k^{2}\left(R_{t} \hat{\theta}-R_{c} \hat{\Theta}\right),}
\end{gathered}
$$

and in the mushy layer as

$$
\begin{gathered}
\left(\mathrm{D}^{2}+\mathrm{D}-\omega-k^{2}\right) \hat{\theta}+\mathscr{F}(\mathrm{D}-\omega) \hat{\chi}=\theta_{b}^{\prime} \hat{w}, \\
{\left[\chi_{b}(\mathrm{D}-\omega)+\chi_{b}^{\prime}\right] \hat{\theta}+\left[\left(\theta_{b}-\mathscr{C}\right)(\mathrm{D}-\omega)+\theta_{b}^{\prime}\right] \hat{\chi}=\theta_{b}^{\prime} \hat{w},} \\
\left(\mathrm{D}^{2}-k^{2}\right) \hat{w}=k^{2} R_{m} \hat{\theta} .
\end{gathered}
$$

The boundary conditions at $z=0$ are

$$
\hat{\theta}=0, \quad \hat{w}=0,
$$


at $z=h_{b}$ are

$$
\begin{gathered}
\hat{\theta}=\hat{\Theta}, \quad \mathrm{D}(\hat{\Theta}-\hat{\theta})=\left(\frac{1-\varepsilon}{\varepsilon}\right) \theta_{b}^{\prime} \hat{h}, \quad[\hat{\theta}]=0 \\
{[\mathrm{D} \hat{\theta}]=\left(\frac{\mathscr{F} \theta_{b}^{\prime}}{\theta_{i}-\mathscr{C}}\right) \hat{h}, \quad \hat{\chi}=\left(\frac{\theta_{b}^{\prime}}{\theta_{i}-\mathscr{C}}\right) \hat{h}, \quad[\hat{w}]=\mathrm{i} k \bar{u}_{b+} \hat{h},} \\
\mathrm{D}^{2} \hat{w}_{+}=\Lambda \sqrt{\frac{\mathscr{H}}{\Pi(1)}}\left(\mathrm{D} \hat{w}_{+}-\mathrm{D} \hat{w}_{-}-\mathrm{i} k \bar{u}_{b+}^{\prime} \hat{h}\right)+\mathrm{i} k \bar{u}_{b+}^{\prime \prime} \hat{h} \\
\left\{\left(\mathrm{D}^{2}+\frac{\mathrm{D}-\omega}{\sigma}-3 k^{2}-\frac{\mathrm{i} k \bar{u}_{b}}{\sigma}\right) \mathrm{D}+\frac{\mathrm{i} k \bar{u}_{b+}^{\prime}}{\sigma}\right\} \hat{w}_{+}+2 \mathrm{i} k^{3} \bar{u}_{b+}^{\prime} \hat{h}+\frac{\mathscr{H}}{\Pi(1)} \mathrm{D} \hat{w}_{-}=0 \\
\text { and at } z \rightarrow \infty \text { are } \quad \hat{\theta} \rightarrow 0, \quad \hat{\Theta} \rightarrow 0, \quad \hat{w} \rightarrow 0, \quad \mathrm{D} \hat{w} \rightarrow 0
\end{gathered}
$$

in which $\mathrm{D} \equiv \mathrm{d} / \mathrm{d} z$.

Equations (20) to (24) constitute a complex eigenvalue problem

$$
F=F\left(R_{m}, k, \omega ; U_{\infty}^{\prime}, \sigma, \varepsilon, \mathscr{F}, \mathscr{C}, \mathscr{H}, \mathscr{A}, \theta_{0}\right) .
$$

The number of eigenvalues $\omega$ of (25) is infinite, as is the number of neutral curves corresponding to $\omega_{r}=0$. Physically, we need only to seek the branch of lowest $R_{m}$, below which $\left(\omega_{r}<0\right)$ the system is stable to small disturbances and above which $\left(\omega_{r}>0\right)$ the system is unstable. Previous studies (Worster 1992; Chen et al. 1994) have shown that, without the imposed shear flow, there are two branches of the neutral curve of lowest $R_{m}$ corresponding respectively to the mushy-layer mode and the boundary-layer mode. With the imposed shear flow, as we shall show later, there are two other branches of neutral curves corresponding to the morphological mode and the suction-layer mode. All these neutral curves are to be examined by the shooting technique along with the orthonormalization process. The numerical procedure is the same as that shown in Chen et al. (1994), to which the reader may refer for details. It is noted that the differential equations become stiffer as $U_{\infty}^{\prime}$ increases, leading to a slower convergent rate of iteration. To improve the convergence efficiency one can rescale the equations by using $U_{\infty}^{\prime}$ as the characteristic velocity to replace $V$ (as will be shown in §6). By and large, it requires about 2000 CPU seconds in the CRAY-YMP/EL of our institute to compute a point of complex eigenvalue.

\section{Effects of imposed shear flow on the stability}

Consider the $26 \mathrm{wt} \%$ ammonium chloride solution whose physical properties are listed in table 1 . We investigate the effect of shear flow on the stability of the present system and therefore will focus on the influence of the parameter $U_{\infty}^{\prime}$. The effects of other physical parameters have been discussed extensively by Chen et al. (1994).

Before proceeding to the analysis, note that in the present system there are three instability modes of different roll-axis orientations: the longitudinal mode whose roll-axis is parallel to the imposed shear flow (corresponding to the case $U_{\infty}^{\prime}=0$ ); the transverse mode whose roll-axis is perpendicular to the imposed shear flow (corresponding to the case $U_{\infty}^{\prime}=U_{\infty}$ ); and the oblique mode whose roll-axis is oblique to the imposed shear flow (corresponding to the case $0<U_{\infty}^{\prime}<U_{\infty}$ ).

We first consider the case $U_{\infty}^{\prime}=0$ (but $U_{\infty}$ may not vanish, see definition (19)). Since in this case $\bar{u}_{b}=0,(20)$ to (24) can be reduced to those of Chen et al. (1994) 

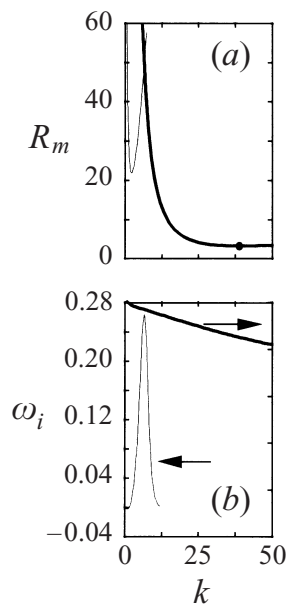
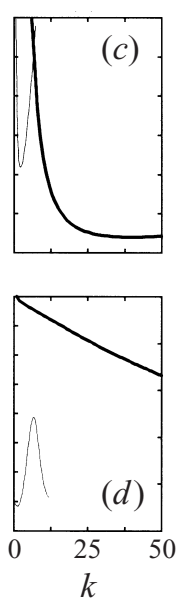
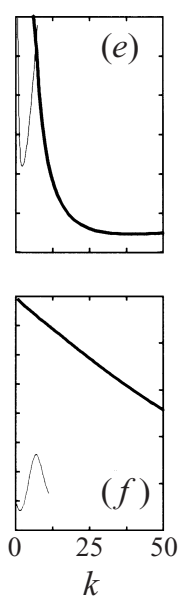
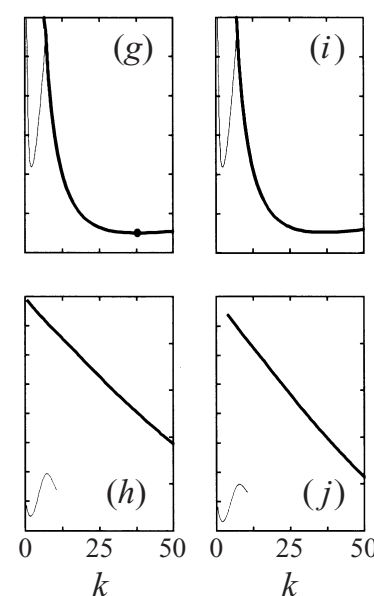
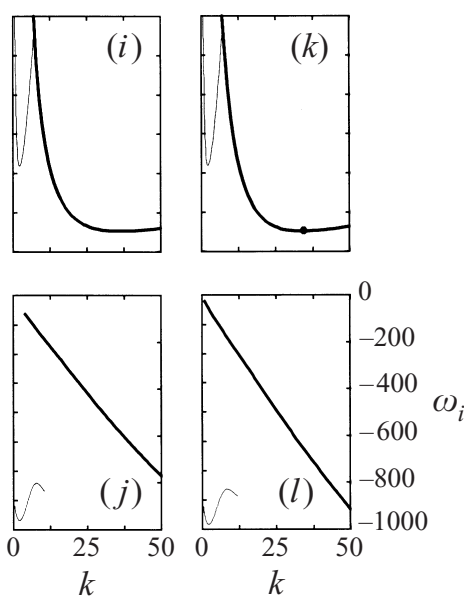

Figure 3. $(a),(c),(e),(g),(i),(k)$ The evolution of the neutral curves of the mushy-layer mode (thin curve) and the boundary-layer mode (thick curve) as the intensity of the imposed shear flow (or the propagation direction of disturbance) changes; $(b),(d),(f),(h),(j),(l)$ The corresponding oscillation frequency of the instability modes $(a, b) U_{\infty}^{\prime}=1000 ;(c, d) U_{\infty}^{\prime}=2000 ;(e, f) U_{\infty}^{\prime}=3000$; $(g, h) U_{\infty}^{\prime}=4000 ;(i, j) U_{\infty}^{\prime}=5000 ;(k, l) U_{\infty}^{\prime}=6000$. Note that the boundary-layer mode is invariably more unstable and of higher oscillation frequency than the mushy-layer mode. Both modes are oscillatory in nature. The stability of the boundary-layer mode increases with $U_{\infty}^{\prime}$ while the mushy-layer mode retains its stability as $U_{\infty}^{\prime}$ changes. All these modes are either oblique $\left(U_{\infty}^{\prime}<U_{\infty}\right)$ or transverse $\left(U_{\infty}^{\prime}=U_{\infty}\right)$.

so that the results of Chen et al. apply. Namely there are three different kinds of instability modes corresponding to the three neutral curves: the mushy-layer mode, the boundary-layer mode, and the oscillatory mode. All these modes are longitudinal.

For $U_{\infty}^{\prime}>0$, the evolution of the neutral curves as $U_{\infty}^{\prime}$ increases from 1000 to 6000 is shown in figure 3, in which the thick curve accounts for the boundary-layer mode and the thin curve accounts for the mushy-layer mode. Results indicate that on applying $U_{\infty}^{\prime}>0$, the oscillatory mode vanishes and the other two modes, which are originally stationary, turn immediately into oscillatory ones, being similar to those found by Linden (1974) and Coriell et al. (1984). The plots in the lower array of figure 3 show the oscillation frequency corresponding to the counterpart of the upper array. The frequency of the critical boundary-layer mode is $O\left(10^{2}\right)$ and that of the critical mushy-layer mode is $O\left(10^{-2}\right)$. Both are negative, implying that the instability modes are the travelling waves propagating along with the shear flow. The results of figure 3 also show that the boundary-layer mode becomes more stable as $U_{\infty}^{\prime}$ increases while the mushy-layer mode is virtually unaffected by the change of $U_{\infty}^{\prime}$. It is also seen that, as $U_{\infty}^{\prime}$ increases, the absolute values of the frequency of both modes also increase, implying that the speed of the travelling wave increases with the shear flow.

As $U_{\infty}^{\prime}$ increases to 7000 (figure $4 a$ ), a closed neutral curve appears near the bottom. The enclosed region becomes larger as $U_{\infty}^{\prime}$ increases (figure $4 c$ ) until, at $U_{\infty}^{\prime}=8158$ (figure $4 e$ ), the closed curve coalesces with the curve of the boundary-layer mode, forming another neutral curve as shown in figures $4(\mathrm{~g})$ and $4(i)$. An enlargement of the evolution of the closed neutral curves is illustrated in figure 5. The closed neutral curve first appears at about $U_{\infty}^{\prime}=6760$ and the enclosed region lies within the domain of $R_{m}<0$. Note that the imposed flow for $U_{\infty}^{\prime}=6760$ is equivalent to a flow of velocity about $1 \mathrm{~cm} \mathrm{~s}^{-1}$ for either $26 \mathrm{wt} \%$ ammonium chloride solution or 

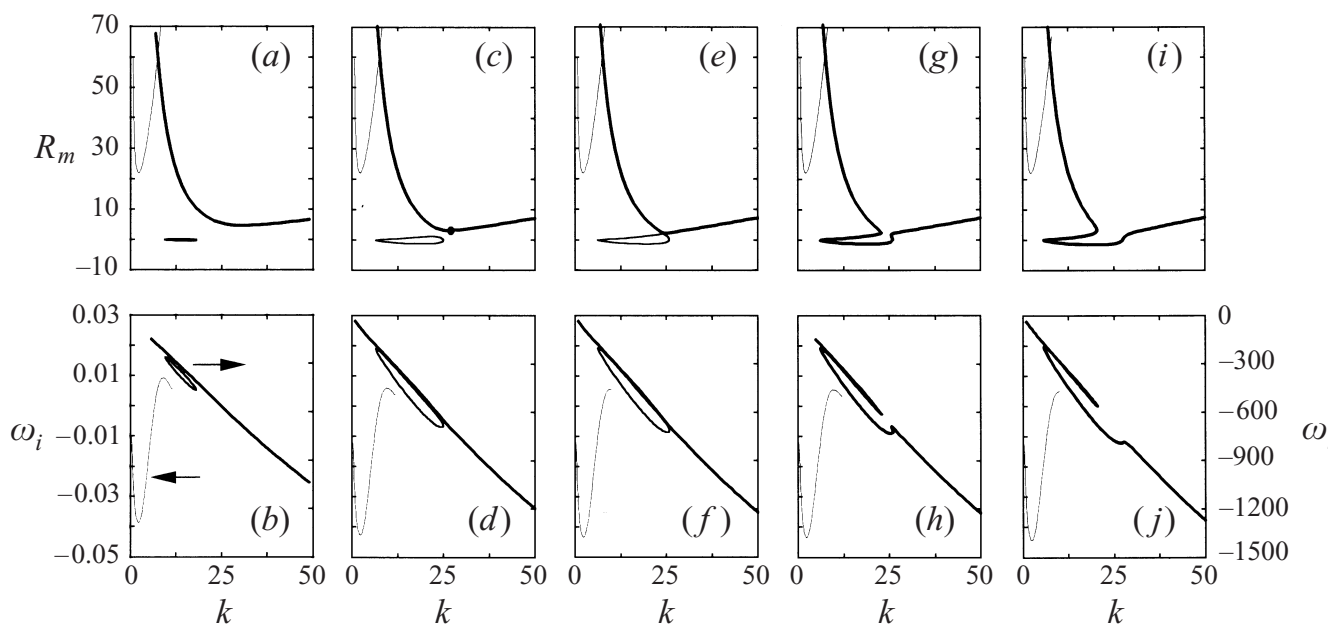

FIGURE 4. As figure 3 but for larger $U_{\infty}^{\prime}:(a, b) U_{\infty}^{\prime}=7000 ;(c, d) U_{\infty}^{\prime}=8000 ;(e, f) U_{\infty}^{\prime}=8158$; $(g, h) U_{\infty}^{\prime}=8200 ;(i, j) U_{\infty}^{\prime}=8500$. The critical $R_{m}$ of the boundary-layer mode decreases with increasing $U_{\infty}^{\prime}$. The neutral curve of the boundary-layer mode moves downwards as $U_{\infty}^{\prime}$ increases, and coalesces with the closed neutral curve of the morphological mode. Similar coalition occurs for the frequency curves of these two modes. The frequencies (and the wave speed) of the boundary layer mode and the morphological mode are of the same order of magnitude.

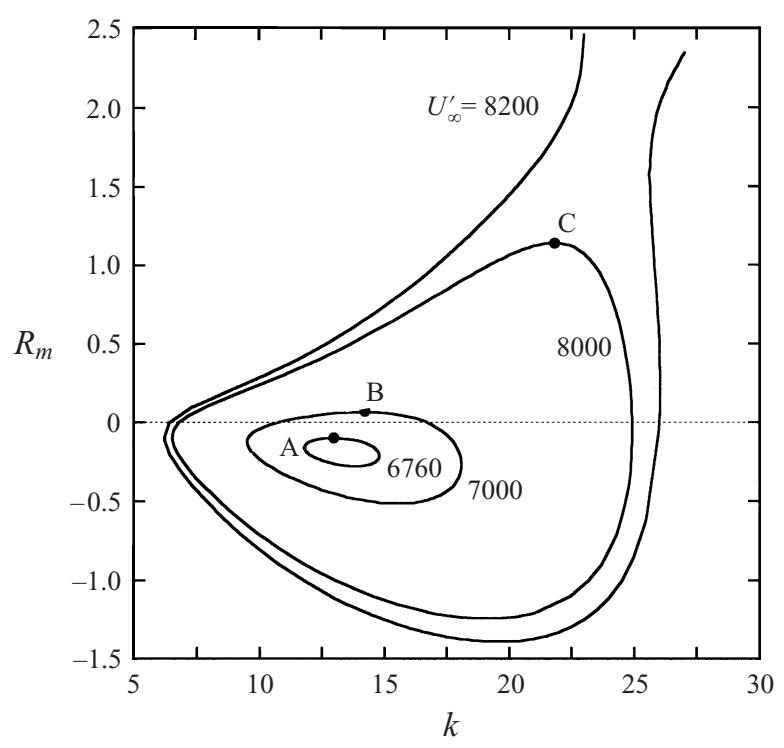

FIGURE 5. An enlargement of the closed neutral curves corresponding to the morphological mode. As $U_{\infty}^{\prime}$ increases, the area of the closed neutral curve increases and it eventually coalesces with the neutral curve of the boundary-layer mode. The three points $\mathrm{A}, \mathrm{B}$, and $\mathrm{C}$ correspond respectively to the cases shown in figures $8(a), 8(b)$ and $8(c)$.

typical sea-ice solution, far below the typical far-field value of $10 \mathrm{~cm} \mathrm{~s}^{-1}$ in sea-ice case suggested by Feltham \& Worster (1999). The area of the enclosed region increases with $U_{\infty}^{\prime}$, gradually becoming a region covering both positive and negative $R_{m}$. The enclosed region covers instability modes of smaller $R_{m}$ than those of the other two modes, implying that they have taken over the role played previously by the boundary- 

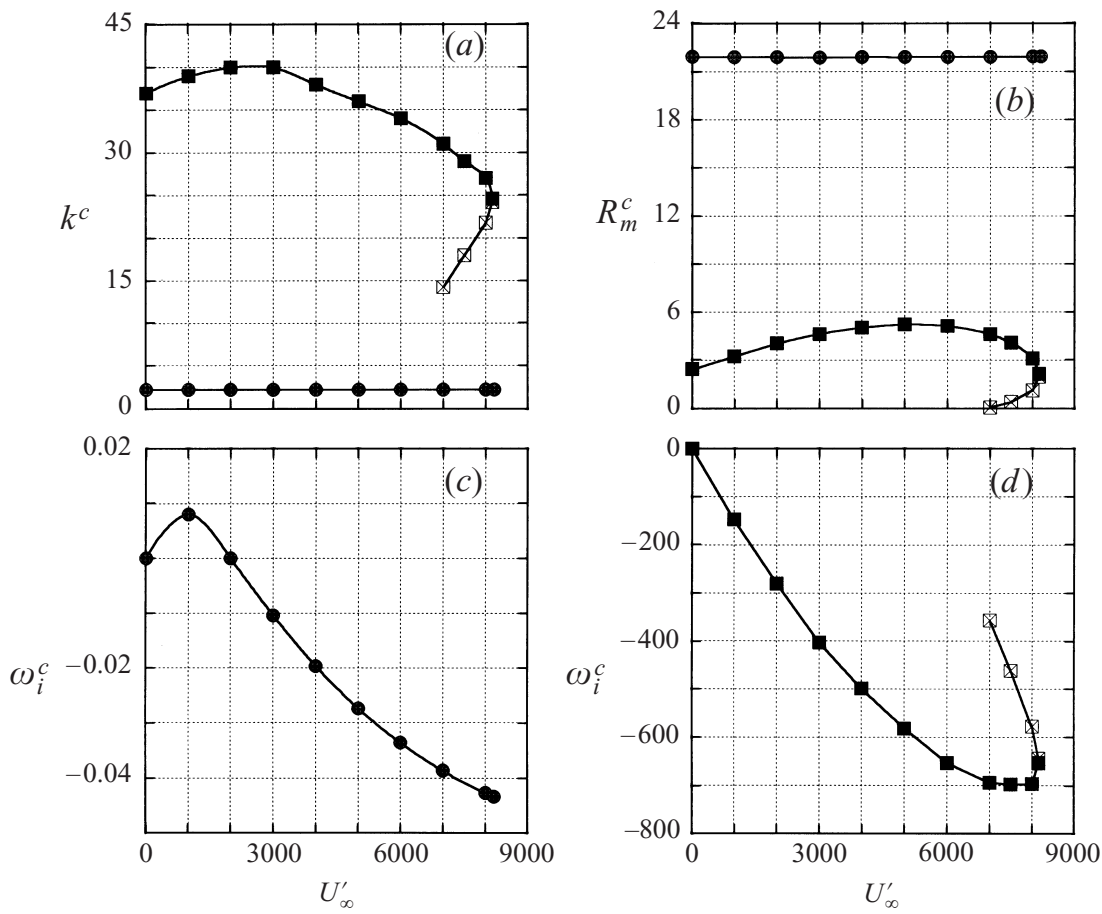

FIGURE 6. Variations of the stability characteristic values with $U_{\infty}^{\prime}$. (a) The critical wavenumber $k^{c}$; (b) the critical Rayleigh number $R_{m}^{c} ;(c)$ the oscillation frequency of the critical mushy-layer mode $\omega_{i}^{c} ;(d)$ the oscillation frequencies of the critical boundary-layer mode and the morphological mode $\omega_{i}^{c}$. $\mathbf{\square}$, The boundary-layer mode; $\otimes$, the morphological mode; $\bullet$, the mushy-layer mode.

layer mode to dominate the system. We call these modes the morphological mode because, as we shall show in $\S 7$, they are the result of the interaction between the imposed shear flow and the interface deformation. Note also that the frequency of the morphological mode is of the same order of magnitude as that of the boundary-layer mode, suggesting that these two modes are of similar travelling speed (see the lower array of figure 4). Note also that negative $R_{m}$ means that the system can be unstable even though the destabilizing solute gradient has become a stabilizing one.

We summarize in figure 6 the variations of the critical $R_{m}$ (or $R_{m}^{c}$ ) and the critical $k$ (or $k^{c}$ ) of both the mushy-layer and boundary-layer modes with $U_{\infty}^{\prime}$, and the corresponding oscillation frequencies. It is seen that the mushy-layer mode, in terms of either $R_{m}^{c}$ or $k^{c}$, is virtually unaffected by the shear flow because the buoyancy force in the mush is much larger than the Bernoulli force (Feltham \& Worster 1999) induced by the imposed flow. The $R_{m}^{c}$ of the boundary-layer mode, nevertheless, increases first with $U_{\infty}^{\prime}$ but then starts to decrease at about $U_{\infty}^{\prime}=5500$. Also shown in figure $6(b)$ is the $R_{m}$ corresponding to the three solid circles of figure 5 , or the maximum $R_{m}$ of the closed neutral curve corresponding to the morphological mode. It is found that the maximum $R_{m}$ eventually coincides with the $R_{m}^{c}$ of the boundary-layer mode as $U_{\infty}^{\prime}$ changes, indicating that the boundary-layer mode and the morphological mode will eventually merge and become the same mode. The $\omega_{i}$ values of these three modes are invariably negative except in a small range $U_{\infty}^{\prime}<2000$ where the $\omega_{i}$ of the mushy-layer mode is slightly larger than zero. The absolute value of $\omega_{i}$ increases with $U_{\infty}^{\prime}$ before the onset of the morphological mode is triggered, i.e. the instability modes 
appear as travelling waves, propagating along with the shear flow with a larger speed as $U_{\infty}^{\prime}$ increases.

The physical meaning of the result shown in figure $6(b)$ is worth examining. It is seen from (19) that $U_{\infty}^{\prime} \leqslant U_{\infty}$, implying that when a driving flow of $U_{\infty}$ is applied in the far field, all the cases $U_{\infty}^{\prime} \leqslant U_{\infty}$ can occur. For example, in an experiment imposing a flow of $U_{\infty}=4000$, the possible $R_{m}^{c}$ can cover a range corresponding to $U_{\infty}^{\prime} \leqslant 4000$. As one can see from figure $6(b)$, the smallest $R_{m}^{c}$ for these cases is the boundary-layer mode corresponding to $U_{\infty}^{\prime}=0$, implying that the longitudinal boundary-layer mode is the most unstable one. This result applies to all the cases before the onset of the morphological mode, which first occurs approximately at $U_{\infty}^{\prime} \approx 7000$. To say this more specifically, before the onset of the transverse morphological mode, the longitudinal boundary-layer mode $\left(U_{\infty}^{\prime}=0\right)$ is invariably the most critical one, while the modes will transverse component $\left(U_{\infty}^{\prime}>0\right)$ are always of larger $R_{m}^{c}$, being unlikely to appear in the system with small disturbances. Namely, as soon as the flow is imposed (no matter how small the flow is), the roll-axis of the boundary-layer mode (a salt-finger type cell) will turn to the direction of the flow, and it becomes a longitudinal mode. This is similar to the experimental findings of Linden (1974) and Chen \& Chen (1997) and to the theoretical result of Coriell et al. (1984).

Although the non-longitudinal mode is unlikely to occur before the onset of the morphological instability, it is nevertheless interesting to illustrate in figure 7, the critical flow patterns of both the mushy-layer mode and the boundary-layer mode corresponding to the cases $U_{\infty}^{\prime}=1000$ to 8000 . All modes are either oblique $\left(U_{\infty}^{\prime}<U_{\infty}\right)$ or transverse $\left(U_{\infty}^{\prime}=U_{\infty}\right)$ and the effective shear flow (the component of shear flow that affects the stability) is in the direction pointing to the right of the page. It is seen that the convection in the mush is hardly influenced by the shear flow while the convection cells in the melt respond to the shear by tilting upstream and propagating downstream.

We also examine the flow patterns of the morphological mode (figure 8) corresponding to the three cases shown by the three solid circles of figure 5. All the modes shown are either oblique or transverse, although the transverse mode is the most critical one. For $U_{\infty}^{\prime}=6760$ (figure $8 a$ ), the morphological mode occurs above the melt/mush interface and the cells tilt in the direction of the shear flow. Its characteristic length is larger than the thickness of the solute boundary layer while smaller than the height of the mush. As $U_{\infty}^{\prime}$ increases to $U_{\infty}^{\prime}=7000$ (figure $8 b$ ) and $U_{\infty}^{\prime}=8000$ (figure $8 c$ ), the cell becomes smaller and tilts gradually against the shear flow. Note that at $U_{\infty}^{\prime}=8158$ the curves of the morphological mode and the boundary-layer mode coalesce (see figure $4 c$ ), and the critical flow patterns of these two modes will have the same characteristics, both tilting to the direction opposite to the shear flow.

\section{Comparison with asymptotic suction flow over fixed porous media}

The results of $\S 5$ show that the morphological mode exists even when buoyancy is absent. Besides, since the melt/mush interface moves vertically upwards with a constant velocity $V$, as the coordinate is fixed on the interface the melt is seen to pass partially vertically downwards into the interface with a constant velocity $V$. Both these facts raise the question of whether the morphological mode is related to the instability of the boundary-layer flow under asymptotic suction, as investigated by Hocking (1975). We attack this question in this section, by focusing on the possible instability mode relevant to the asymptotic suction effect.

Based on the characteristic scales used in the present analysis, the critical wavenum- 

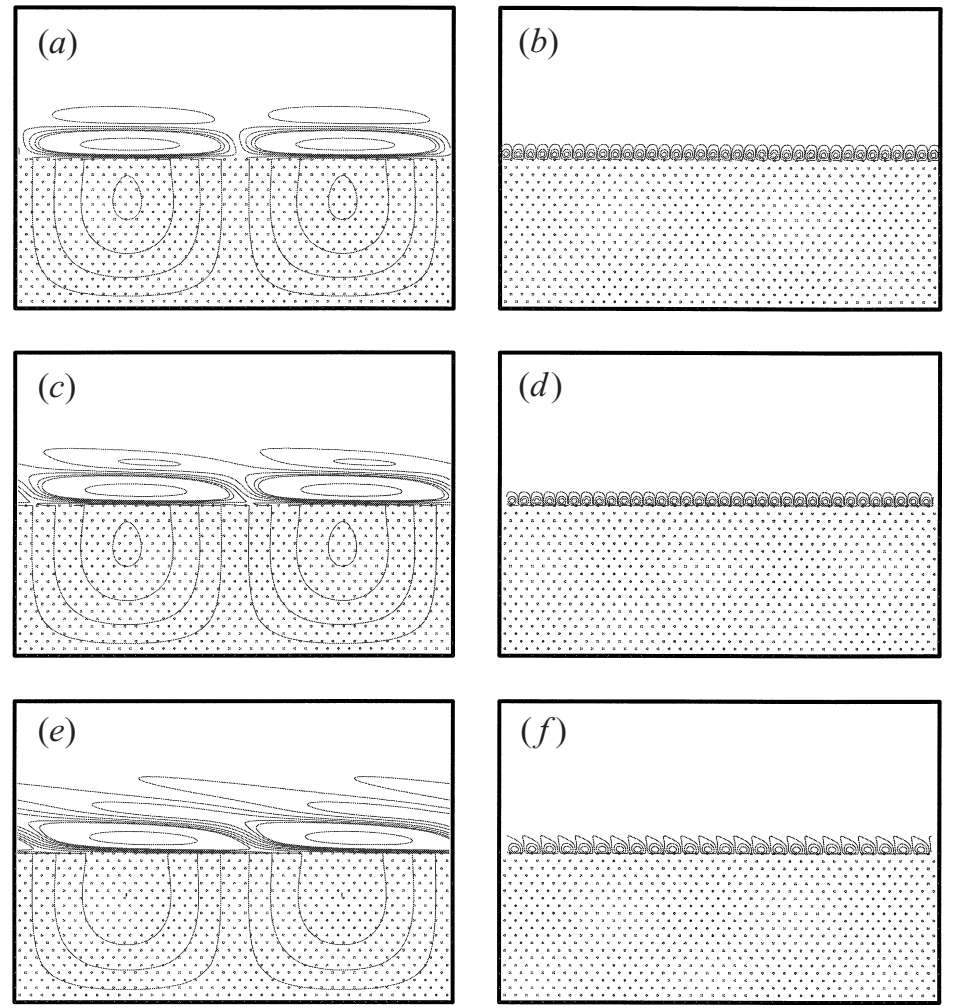

FiguRE 7. The critical flow patterns correspond to the cases of figures $3(a), 3(\mathrm{~g})$ and $4(c)$. $(a)$ The mushy-layer mode for $U_{\infty}^{\prime}=1000$, its critical values are $k^{c}=2.2, R_{m}^{c}=21.921$. (b) The boundary-layer mode for $U_{\infty}^{\prime}=1000$, for which $k^{c}=39, R_{m}^{c}=3.227$. (c) The mushy-layer mode for $U_{\infty}^{\prime}=4000$, for which $k^{c}=2.2, R_{m}^{c}=21.901$. (d) The boundary-layer mode for $U_{\infty}^{\prime}=4000$ for which $k^{c}=38, R_{m}^{c}=5.022$. (e) The mushy-layer mode for $U_{\infty}^{\prime}=8000$, for which $k^{c}=2.2$, $R_{m}^{c}=21.937$. (f) The boundary-layer mode for $U_{\infty}^{\prime}=8000$, for which $k^{c}=27, R_{m}^{c}=3.080$. In general, the flow patterns in the mush are barely influenced by the imposed shear flow, which moves towards the right of the figure. The convection cells in the melt, either of the mushy-layer mode or of the boundary-layer mode, respond to the imposed shear with a tilt-distortion of their shape in the opposite direction to that of the imposed flow. All the flow patterns shown here are either oblique $\left(U_{\infty}^{\prime}<U_{\infty}\right)$ or transverse $\left(U_{\infty}^{\prime}=U_{\infty}\right)$. Note that the height of the mushy layer (the dotted region) is 0.991 , about the same order of magnitude as the thermal boundary layer thickness, which is unity. The solute boundary layer above the melt/mush interface is of the order of the inverse Lewis number $\varepsilon$, i.e. $O(0.013)$.

ber of the instability mode can be as small as $O\left(10^{-2}\right)$ and the critical shear flow velocity can be as high as $U_{\infty}^{\prime}=50000$. It turns out that, under such a scaling, the convergence of the numerical computation is rather slow and in most of cases the iteration fails to converge. We therefore need to change the present scale system, which was defined on the basis of the thermal and solute gradients in previous sections, into the one which can reflect the characteristics of the shear flow instability under asymptotic suction. Accordingly, based on the shear flow velocity and the kinematic viscosity, we choose $U_{\infty}^{\prime}$ for velocity, $v / V$ for length, $v / V U_{\infty}^{\prime}$ for time, and the other scales remain the same as before, and also assume $U_{\infty}^{\prime} \neq 0$ to consider all the modes with a transverse component. Note that the scales in the mushy layer do not change, so that (21) remains the same. The disturbance equations in the fluid 

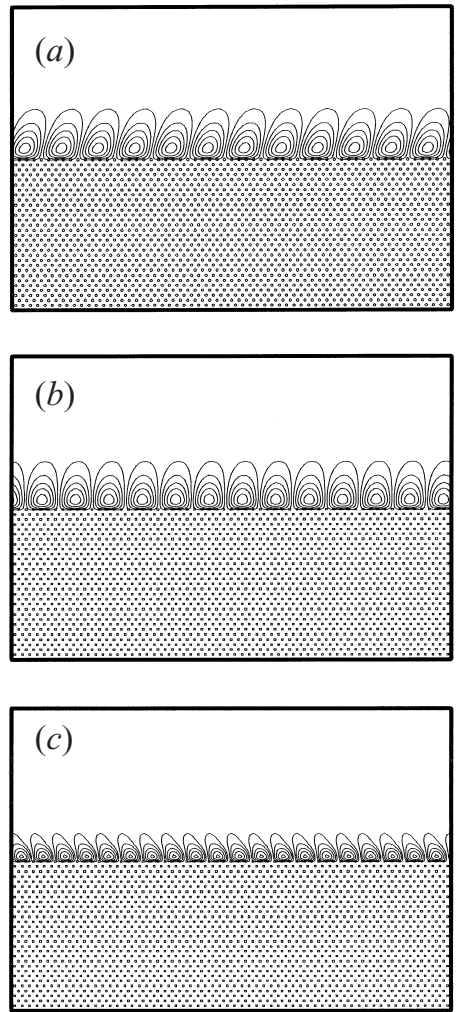

FiguRE 8 . The convection cells of the morphological mode corresponding to the three solid points shown in figure 5. (a) $U_{\infty}^{\prime}=6760, k^{c}=13, R_{m}^{c}=-0.0987 ;(b) U_{\infty}^{\prime}=7000, k^{c}=14.25, R_{m}^{c}=0.0666$; (c) $U_{\infty}^{\prime}=8000, k^{c}=21.8, R_{m}^{c}=1.139$. In $(a)$ and $(b)$ the morphological mode is isolated from the boundary-layer mode, and is tilted into the direction of the imposed flow. In $(c)$, the morphological mode merges with the boundary-layer mode, the convection cell tilts into the opposite direction to that of the imposed flow, being the same as the boundary-layer mode. All the flow patterns shown here are either oblique $\left(U_{\infty}^{\prime}<U_{\infty}\right)$ or transverse $\left(U_{\infty}^{\prime}=U_{\infty}\right)$. Note that the height of the mushy layer (dotted region) is 0.991 , about the same order of magnitude of the thermal boundary layer thickness, which is unity. The solute boundary layer above the melt/mush interface is of the order of the inverse Lewis number $\varepsilon$, i.e. $O(0.013)$.

layer are changed into the following forms:

$$
\begin{gathered}
\left(\mathrm{D}^{2}+\sigma \mathrm{D}^{*}-\mathrm{i} \sigma k^{*} \bar{u}_{b}-\sigma R e^{\prime} \omega^{*}-k^{* 2}\right) \hat{\theta}=\sigma R e^{\prime} \frac{\mathrm{d} \theta_{b}}{\mathrm{~d} z^{*}} \hat{w}^{*}, \\
\left(\varepsilon \mathrm{D}^{* 2}+\sigma \mathrm{D}^{*}-\mathrm{i} \sigma k^{*} \bar{u}_{b}-\sigma R e^{\prime} \omega^{*}-\varepsilon k^{* 2}\right) \hat{\Theta}=\sigma R e^{\prime} \frac{\mathrm{d} \Theta_{b}}{\mathrm{~d} z^{*}} \hat{w}^{*}, \\
{\left[\mathrm{D}^{* 2}+\left(\mathrm{D}^{*}-R e^{\prime} \omega^{*}\right)-k^{* 2}-\mathrm{i} k^{*} \bar{u}_{b}\right]\left(\mathrm{D}^{* 2}-k^{* 2}\right) \hat{w}^{*}+\mathrm{i} k^{*} \frac{\mathrm{d}^{2} \bar{u}_{b}}{\mathrm{~d} z^{* 2}} \hat{w}^{*}=\frac{\sigma^{2} k^{* 2}}{R e^{\prime}}\left(R_{t} \hat{\theta}-R_{c} \hat{\Theta}\right),}
\end{gathered}
$$

in which

$$
R e^{\prime}=\operatorname{Re} \frac{k_{x}}{k}=\frac{\left(U_{\infty} V\right)(v / V)}{v} \cos \left(\phi_{k}\right)=U_{\infty}^{\prime},
$$

is the reduced Reynolds number, resulting from Squire's transformation. We seek the critical value of $R e^{\prime}$. In (2.6) and (2.7), $\mathrm{D}=\mathrm{d} / \mathrm{d} z^{*}, z^{*}=z / \sigma$ is the new dimension- 

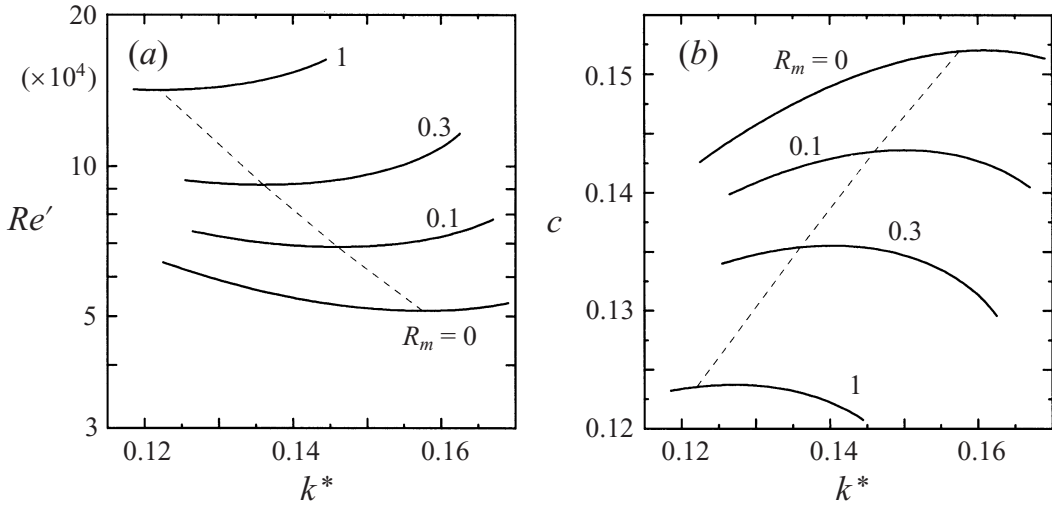

FIGURE 9. The neutral curves $(a)$ and the propagating wave speed $(b)$ of the suction-layer modes. The suction-layer mode is of a length scale close to the thickness of the imposed shear boundary layer, which is about 50 times larger than the height of the mushy layer. The suction-layer mode is much more stable than either the morphological mode or the boundary-layer mode or the mushy-layer mode, and is therefore not likely to occur in the present system. The suction-layer mode becomes more stable when the solidification effect (as shown by $R_{m}$ ) is more intense.

less length, $k^{*}=\sigma k$ the new dimensionless wavenumber, $\omega^{*}=\left(\sigma / U_{\infty}^{\prime}\right) \omega$ the new dimensionless frequency, and $\hat{w}^{*}=\hat{w} / U_{\infty}^{\prime}$ the new dimensionless disturbance velocity.

The new boundary conditions at $z^{*} \rightarrow \infty$ are

$$
\hat{\theta} \rightarrow 0, \quad \hat{\Theta} \rightarrow 0, \quad \hat{w}^{*} \rightarrow 0, \quad \mathrm{D}^{*} \hat{w}^{*} \rightarrow 0,
$$

and at the interface $z^{*}=h_{b} / \sigma\left(\right.$ or $\left.z=h_{b}\right)$ are

$$
\begin{gathered}
\hat{\theta}=\hat{\Theta}, \quad \mathrm{D}^{*}(\hat{\Theta}-\hat{\theta})=\sigma\left(\frac{1-\varepsilon}{\varepsilon}\right)\left(\frac{\mathrm{d} \theta_{b}}{\mathrm{~d} z^{*}}\right) \hat{h}^{*} \\
\hat{\theta}]=0, \quad \mathrm{D}^{*} \hat{\theta}_{+}-\sigma \mathrm{D} \hat{\theta}_{-}=\left(\frac{\sigma \mathscr{F}\left(\mathrm{d} \theta_{b} / \mathrm{d} z^{*}\right)}{\theta_{i}-\mathscr{C}}\right) \hat{h}^{*} \\
\hat{\chi}=\left(\frac{\mathrm{d} \theta_{b} / \mathrm{d} z^{*}}{\theta_{i}-\mathscr{C}}\right) \hat{h}^{*}, \quad R e^{\prime} \hat{w}_{+}^{*}-\hat{w}_{-}=\mathrm{i} k^{*} \bar{u}_{b+} \hat{h}^{*} \\
R e^{\prime} \mathrm{D}^{* 2} \hat{w}_{+}^{*}=\sigma \Lambda \sqrt{\frac{\mathscr{H}}{\Pi(1)}}\left(R e^{\prime} \mathrm{D}^{*} \hat{w}_{+}^{*}-\sigma \mathrm{D} \hat{w}_{-}-\mathrm{i} k^{*}\left(\frac{\mathrm{d} \bar{u}_{b}}{\mathrm{~d} z^{*}}\right)_{+} \hat{h}^{*}\right)+\mathrm{i} k^{*}\left(\frac{\mathrm{d}^{2} \bar{u}_{b}}{\mathrm{~d} z^{* 2}}\right)_{+} \hat{h}^{*} \\
R e^{\prime}\left[\left(\mathrm{D}^{* 2}+\mathrm{D}^{*}-R e^{\prime} \omega^{*}-3 k^{* 2}-\mathrm{i} k^{*} \bar{u}_{b}\right) \mathrm{D}^{*}+\mathrm{i} k^{*}\left(\frac{\mathrm{d} \bar{u}_{b}}{\mathrm{~d} z^{*}}\right)_{+}\right] \hat{w}_{+}^{*} \\
+2 \mathrm{i} k^{* 3}\left(\frac{\mathrm{d} \bar{u}_{b}}{\mathrm{~d} z^{*}}\right)_{+} \hat{h}^{*}+\frac{\sigma^{3} \mathscr{H}}{\Pi(1)} \mathrm{D} \hat{w}=0
\end{gathered}
$$

in which $\hat{h}^{*}=\hat{h} / \sigma$ is the new dimensionless disturbance of the interface position. The boundary conditions at the mush/solid interface remains as in (22). Equations (26) and (21) together with the boundary conditions (22), (28) and (29) constitute again a complex eigenvalue problem, which is solved with the same numerical scheme used in previous sections.

Figure 9 shows the neutral curves for various $R_{m}$. The critical points corresponding 


\begin{tabular}{crcc}
\hline$R_{m}$ & \multicolumn{1}{c}{$R^{\prime}$} & $k^{*}$ & $c$ \\
0.0 & 51306 & 0.1575 & 0.1519 \\
0.1 & 68886 & 0.1460 & 0.1435 \\
0.3 & 91818 & 0.1360 & 0.1354 \\
1.0 & 141853 & 0.1220 & 0.1235
\end{tabular}

TABLE 2. The characteristic values of the critical points of the neutral curves shown in figure 9 .

to the minimum of $R e^{\prime}$ (equivalent to $U_{\infty}^{\prime}$, see (27)) are connected with the dotted curve and the corresponding values of $R e^{\prime}, k^{*}$, and $c=-\omega_{i}^{*} / k^{*}$ (the wave speed) are listed in table 2. It is shown in figure $9(a)$ that the case of $R_{m}=0$, which accounts for the case of zero buoyancy, is most unstable. The critical $R e^{\prime}$ increases with $R_{m}$, implying that the solidification is a stabilizing factor for the shear flow with asymptotic suction. Figure $9(b)$ illustrates that the solidification causes a reduction of the propagation speed of the instability mode, which again is due to the stabilizing thermal field in the system. For the shear layer flow under asymptotic suction of Hocking (1975), we found the critical values are $R e^{\prime}=54370, k^{*}=0.1555, c=0.150$; namely, his $R e^{\prime}$ is much larger than the present case while $k^{*}$ amd $c$ are quite close. The less stable situation of the present system is a result of the melt/mush interface being allowed to deform while that of Hocking is a fixed porous boundary. A free-to-deform boundary leads naturally to a less stable flow. Since this instability mode is of the length scale of the shear boundary-layer thickness (see figure 2) and its characteristics are closely related to the suction effect, we call this mode the suction-layer mode.

In summary, the morphological mode and the suction-layer mode have very different length scales and different stability criteria, while both of them result directly from the imposition of the shear flow. The morphological mode occurs in a shallow region above the melt/mush interface while the suction-layer mode occurs in a region covering virtually the whole depth of the shear boundary layer. The critical $R e^{\prime}$ (or $U_{\infty}^{\prime}$ ) of the morphological mode is much smaller than that of the suction-layer mode, suggesting that the morphological mode dominates over the suction-layer mode in the system. Both modes are transverse, propagating in the direction of the imposed shear.

\section{Mechanism triggering the morphological mode}

In this section, we discuss the mechanism related to the occurrence of the morphological mode. We shall first examine whether the morphological mode is directly related to the deformation of the melt/mush interface, as concluded by Feltham \& Worster (1999). To do this, we assume that the interface remains planar (or nondeformable) under perturbation. Since the height of the mush is now a constant, one of the interface conditions can be omitted and we choose the marginal equilibrium condition $(7 b)$. We implement the linear stability analysis for the case of $U_{\infty}^{\prime}=8200$. The result is shown in figure 10, in which the solid curves correspond to the case with a deformable interface and the dashed curve corresponds to the case with a fixed-planar interface. It is seen that, when the interface is fixed to be planar, the morphological mode disappears and the system becomes more stable. The role played by the interface is thus confirmed; namely, without allowing interface deformation, there is no morphological mode. 


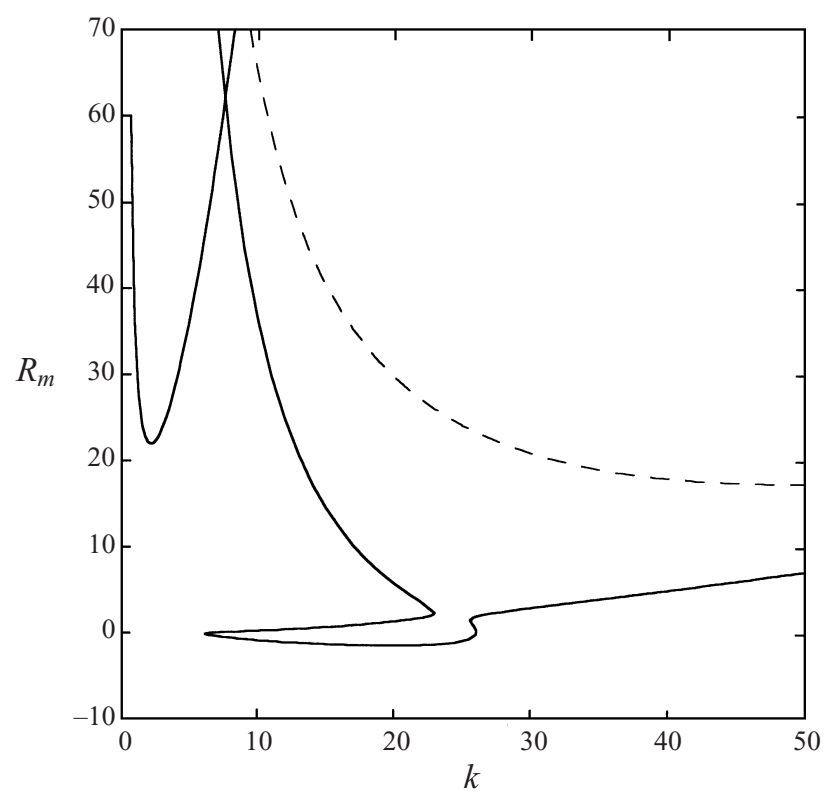

FIGURE 10. The neutral curves for $U_{\infty}^{\prime}=8200$, in which the two solid curves show respectively the mushy-layer mode (left-hand curve) and the boundary-layer mode (right-hand curve). The dashed curve shows the case assuming that the perturbation on the interface to be zero. It is seen that the flow of fixed interface is more stable than the flow allowing interface deformation, and the morphological mode does not exist when the interface is fixed.

To elucidate more clearly the stability mechanism, we also examine the detailed flow structure and the associated temperature distribution. The case we consider is $U_{\infty}^{\prime}=7000$, point B of figure 5. Figure 11(a) illustrates the streamlines of the flow in both the melt and the mush (solid curves) and the flow pattern of the morphological mode (dotted curves). Note that the present morphological mode is the same mode as shown in figure $8(b)$ when the same contour levels are chosen. The streamlines in the melt are a combination of the imposed shear flow and the morphological mode convection. The magnitude of the flow in the mush is only about one-thousandth that in the melt, and therefore named weak flow by Feltham \& Worster (1999).

By comparing figure 11(a) with figure 2(a) of Feltham \& Worster (inviscid flow), we found that the present weak flow is different from theirs by a phase shift. This difference is due to the effect of viscosity in the melt, which introduces a phase lag between the interface corrugation and the pressure variation. The weak flow is no longer impinging on the crest of the corrugated interface, but about halfway between the crest and the trough. This suggests that the weak flow may contribute not only to the deformation of the interface, but also to its translation. This scenario can be explained more clearly by the isotherms shown in figure 11(b). Because of the direction of the weak flow, the heat advection compresses the isotherms in the mush at the upstream face of the trough and rarefy at the downstream face. This implies that the upstream face will solidify but the downstream face will melt because the conduction of heat away from the upstream face is enhanced while that from the downstream face is inhibited. Accordingly, the interface is likely to translate in the direction of the imposed flow. On the other hand, similarly, the perturbed flow in the melt compresses the isotherms at the upstream face of the trough and spreads them at the downstream face. This implies that the upstream face will melt while 
(a)

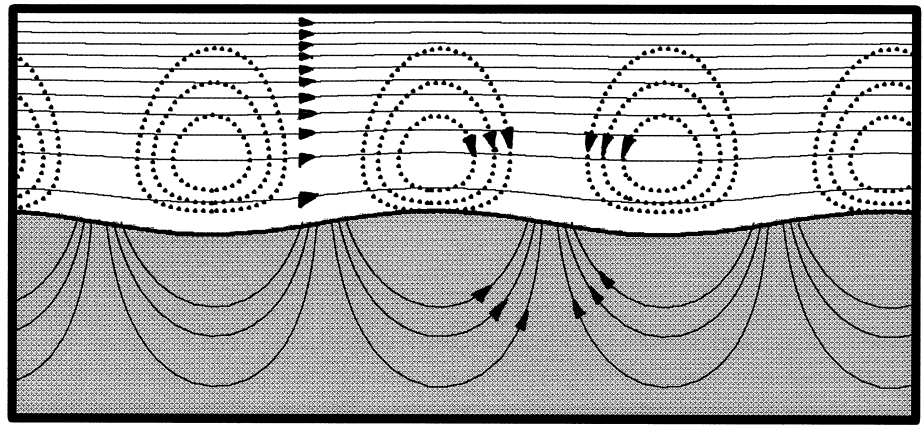

(b)

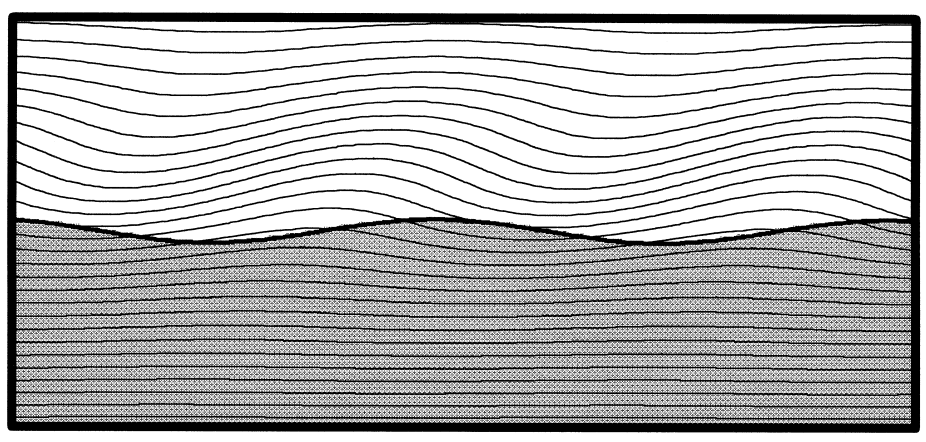

FIGURE 11. The streamline patterns and isotherms corresponding to point $\mathrm{B}$ of figure 5 , a local maximum of the neutral curve. The imposed shear flow moves from left to right. (a) The streamlines in the melt represent a combination of imposed shear flow and the morphological mode shown by the dashed curves. The magnitude of flow perturbation in the mush is one-thousandth that of the perturbed flow in the melt. It is therefore called a weak flow. (b) The isotherms corresponding to the flow shown in $(a)$. The maximum heat flux on the interface occurs at the upstream face of the trough and the minimum occurs at the downstream face.

the downstream face will solidify because the conduction of heat from the upper hot region toward the upstream face is enhanced while that toward the downstream face is suppressed. Therefore, the perturbed flow in the melt is likely to make the interface translate upstream and deform in the opposite sense.

The above conclusion gives rise to another question: Is the weak flow in the mush or the perturbed flow in the melt responsible for the enhancement of the interface deformation? The answer can be seen from figure $6(d)$, where the instability mode is propagating in the direction of the imposed flow (because $\omega_{i}^{c}<0$ ). We thus conclude that the morphological instability is triggered by the weak flow in the mush, and the perturbed flow in the melt plays a stabilizing role. This conclusion is supported by Feltham \& Worster (1999), who asserted that their morphological instability relies on the weak flow in the mush induced by the Bernoulli effect, and the inclusion of the viscosity in the melt makes the instability weaker.

In summary, the imposed parallel flow over an incipiently corrugated interface causes a virtually periodical perturbation of pressure along the interface due to the Bernoulli effect. This then produces a flow perturbation in the melt (the morphological mode) and a weak flow in the mush (due to the large Darcy resistance). Although the perturbed flow in the mush is much weaker than that in the melt, it however plays an 
important role in the positive feedback mechanism of the morphological instability. On the other hand, the perturbed flow in the melt retards the growth of the interface. In other words, the morphological instability is affected by the flow induced in both the mush and the melt, but stimulated primarily by the flow in the mush.

\section{Concluding remarks}

We have investigated the effect of shear flow on the convective stability in a binary solution directionally solidified from below. A $26 \mathrm{wt} \%$ ammonium chloride solution is considered as the working fluid and the horizontal domain is considered to be infinite so that a shear flow parallel to melt/mush interface can be imposed. Results from present analyses show that there are four instability modes of interest in the present system: the mushy-layer mode, the boundary-layer mode, the morphological mode and the suction-layer mode. Because the Bernoulli force is much weaker than the solute buoyancy, the mushy-layer mode is virtually unaffected by the imposed shear and remains more stable than the other instability modes. The boundary-layer mode, on the other hand, senses the influence of the imposed flow as soon as the flow is imposed, responding with the immediate alignment of the cell axis with the direction of the shear flow. As a result, the longitudinal boundary-layer mode dominates the system as the shear flow increases in intensity (as shown by $U_{\infty}$ ) until the onset of the morphological mode, a transverse mode triggered mainly by the pressure variation along the corrugated interface. As $U_{\infty}$ increases, the morphological mode merges with the boundary-layer mode while retaining its original features. The features of this novel morphological mode are summarized as follows:

(i) It is a transverse mode.

(ii) Its length scale is larger than the solute boundary layer thickness but smaller than the thermal or the shear boundary layer thickness.

(iii) It appears as a travelling wave, propagating along with the shear flow.

(iv) It can occur even if the destabilizing solute gradient vanishes (i.e. $R_{m}=0$ ), namely the buoyancy plays no role in its formation.

(v) It differs greatly from the suction-layer mode in both the wavelength and the stability criteria.

(vi) It is triggered by the weak flow of the mushy layer, which results in the pressure variation along the corrugated melt-mush interface (the so-called Bernoulli force), being the same mode as revealed by Feltham \& Worster (1999).

Delves $(1968,1971)$ and Coriell et al. (1984) showed that, as the shear flow is imposed upon the melt/solid interface, the modes with a transverse component are invariably inhibited so that the longitudinal mode dominates the system. This is similar to the present result before the morphological mode appears. Nevertheless, we have shown that within a large parameter range the morphological (transverse) mode dominates the system. This difference stems mainly from the existence of the mushy layer, in which a weak flow is induced by the imposed shear flow in the melt. This weak flow enhances the deformation of the melt/mush interface as well as the pressure variation along the interface and then triggers the onset of the morphological mode.

The morphological mode is very unstable, and can be triggered even without buoyancy. The imposed shear flow is the major reason for its formation. Chung \& Chen (2000b) show that there is a strong shear flow above the melt/mush interface in the experiment of Sample \& Hellawell (1984), implying that the morphological mode may dominate the system. We nevertheless note that in their system, owing to inclined rotation the centrifugal force and Coriolis force may play significant roles 
in determining the stability, while in the present system neither of forces exists. This difference may lead to different conclusions, which might merit future study.

The financial support for this research and the post-doctoral fellowship for C.A.C. through the research grant NSC 89-2212-E-002-024 is gratefully appreciated.

\section{REFERENCES}

Amberg, G. \& Homsy, G. M. 1993 Nonlinear analysis of buoyant convection in binary solidification with application to channel formation. J. Fluid Mech. 252, 79-98.

ANDERSON, D. M. \& Worster, M. G. 1995 Weakly nonlinear analysis of convection in a mushy layer during the solidification of binary alloys. J. Fluid Mech. 302, 307-331.

Beavers, G. S. \& Joseph, D. D. 1967 Boundary conditions at a naturally permeable wall. J. Fluid Mech. 30, 199-207.

Chen, C. F. \& Chen, F. 1997 Salt-finger convection generated by lateral heating of a solute gradient. J. Fluid Mech. 352, 161-176.

Chen, F., Lu, J. W. \& Yang, T. L. 1994 Convective instability in ammonium chloride solution directionally solidified from below. J. Fluid Mech. 276, 163-187.

Chung, C. A. 1998 Mechanisms driving the formation of plume convection, PhD dissertation, National Taiwan University, Taipei, Taiwan.

Chung, C. A. \& Chen, F. $2000 a$ Onset of plume convection in the mushy layer. J. Fluid Mech. 408, 53-82.

Chung, C. A. \& Chen, F. $2000 \mathrm{~b}$ Convection in directionally solidifying alloys under inclined rotation. J. Fluid Mech. 412, 93-123.

Copley, S. M., Giamei, A. F., Johnson, S. M. \& Hornbecker, M. F. 1970 The origin of freckles in undirectionally solidified castings. Metall. Trans. 1, 2193-2204.

Coriell, G. B., McFadden, G. B., Boisvert, R. F. \& Sekerka, R. F. 1984 Effect of a forced Couette flow on coupled convective and morphological instabilities during unidirectional solidification. J. Cryst. Growth 69, 15-22.

DAvis, S. H. 1990 Hydrodynamic interactions in directional solidification. J. Fluid Mech. 212, 241-262.

DeLves, R. T. 1968 Theory of stability of a solid-liquid interface during growth from stirred melts. J. Cryst. Growth 3, 562-568.

Delves, R. T. 1971 Theory of the stability of a solid-liquid interface during growth from stirred melts - II. J. Cryst. Growth 8, 13-25.

Feltham, D. L. \& Worster, M. G. 1999 Flow-induced morphological instability of a mushy layer. J. Fluid Mech. 391, 337-357.

Forth, S. A. \& WheEler, A. A. 1989 Hydrodynamic and morphological stability of the unidirectional solidification of a freezing binary alloy: a simple model. J. Fluid Mech. 202, 339-366.

Forth, S. A. \& WheEler, A. A. 1992 Coupled convective and morphological instability in a simple model of the solidification of a binary alloy, including a shear flow. J. Fluid Mech. 236, 61-94.

Gershuni, G. Z. \& ZhukhovitskiI, E. M. 1976 Convective Stability of Incompressible Fluids. Keter Press, Jerusalem.

Hocking, L. M. 1975 Non-linear instability of the asymptotic suction velocity profile. Q. J. Mech. Appl. Maths 28, 341-353.

Linden, P. F. 1974 Salt fingers in a steady shear flow. Geophys. Fluid Dyn. 6, 1-27.

Lu, J. W. \& Chen, F. 1997 Rotation effects on the convection of binary alloys unidirectional solidified from below. Intl J. Heat Mass Transfer 40, 237-246.

McFadden, G. B., Coriell, S. R. \& Alexander, J. I. D. 1988 Hydrodynamic and free boundary instabilities during crystal growth: The effect of a plane stagnation flow. Commun. Pure Appl. Maths 41, 683-706.

Sample, A. K. \& Hellawel, A. 1984 The mechanism of formation and prevention of channel segregation during alloy solidification. Metall. Trans. A 15, 2163-2173.

TAIT, S. \& JAUPART, C. 1992 Compositional convection in a reactive crystalline mush and melt differentiation. J. Geophys. Res. 97(B5), 6735-6759.

Worster, M. G. 1986 Solidification of an alloy from a cooled boundary. J. Fluid Mech. 167, 481-501.

Worster, M. G. 1992 Instabilities of the liquid and mushy regions during solidification of alloys. J. Fluid Mech. 237, 649-669. 